\title{
Frequent association of delayed tear clearance in ocular irritation
}

Pinnita Prabhasawat, Scheffer C G Tseng

\begin{abstract}
Aim-To explore the pathogenic role of delayed tear clearance.

Methods-By comparing 10 patients with punctal obstruction and 20 asymptomatic normals, delayed tear clearance was diagnosed in 70 patients without apparent punctal obstruction using fluorescein clearance test.

Results-The majority were older (71.4 (SD 1.2) years) and women (66\%). Frequent complaints included redness, itching, mucus discharge, and crusting, which tended to be worse upon awakening. Common associated problems were medicamentosa (13\%), drug induced pseudopemphigoid, ocular hypertension (27\%), and glaucoma (7\%). Topical non-preserved $1 \%$ methylprednisolone resulted in subjective $(83 \%)$ and objective $(80 \%)$ improvement and resolution of delayed tear clearance $(87 \%)$.

Conclusion-These results indicate strong association of delayed tear clearance with intrinsically and extrinsically generated ocular surface inflammation. The presence of delayed tear clearance may set up a vicious cycle to aggravate the existing inflammation. Future prospective studies are needed to delineate the pathogenic role of delayed tear clearance in various ocular surface disorders.

(Br f Ophthalmol 1998;82:666-675)
\end{abstract}

The ocular surface is covered by a thin layer of preocular tear film, which is composed of lipids, electrolyte, and protein containing aqueous fluid and mucins. Under normal circumstances, aqueous tears are primarily secreted by the lacrimal gland, spread over the entire ocular surface by lid blinking, and then cleared from the eye into the nose through the nasolacrimal drainage system, which includes superior and inferior puncta and canaliculi, the lacrimal sac, and the nasolacrimal duct. ${ }^{12}$ Therefore, tear functions depend not only on necessary tear components but also on hydrodynamic elements, which include tear secretion (flow), tear volume, and tear clearance or turnover.

We have long recognised that decreased tear secretion (flow) by lacrimal glands results in the disease state of aqueous tear deficiencythat is, keratoconjunctivitis sicca. For tear clearance, we are also familiar with epiphorathat is, excessive constant tearing, caused by anatomical obstruction of the nasolacrimal drainage system leading to frank delay. Nevertheless, it is not clear if subclinical delayed tear clearance is pathogenic. Even if we presume this might be the case, it remains obscure as to what type of ocular surface disorders tend to be more associated with delayed tear clearance, which may aggravate the primary disease.

Clinically, one common way of measuring tear clearance is to judge the speed of disappearance from the ocular surface of exogenously added fluorescein, a method originally used by Nover and Jaeger. ${ }^{3}$ Various methods have been developed and can be grossly subdivided into two major groups. The first group detects how fast the dye appears in the nasal cavity by the use of a cotton tip in the traditional Jones I test, ${ }^{4}$ or by direct visualisation through endoscopy. ${ }^{5}$ The second group measures how slow the dye is retained on the ocular surface by visual semiquantitation, ${ }^{67}$ wetting with Schirmer strips, ${ }^{3}$ or fluorophotometry, ${ }^{8}$ of which the latter can be directed to the marginal $\operatorname{strip}^{89}$ or to the precorneal tear film. ${ }^{10-13}$

Based on the second principle, we employed a modified fluorescein clearance test (FCT) which detected fluorescein retained in serial Schirmer strips over a period of 30 minutes, and have identified a number of patients with subclinical delayed tear clearance. A retrospective review of their clinical data reveals characteristic morbidity. Because of its association with patients' ocular surface problem and its resolution by non-preserved methylprednisolone leading to clinical improvement, we propose that this strong association warrants future studies to delineate whether delayed tear clearance is indeed pathogenic.

\section{Methods}

PATIENTS

From a total of 2147 FCT test results, performed from 1986 to 1995 at the Bascom Palmer Eye Institute as part of the routine examination for patients complaining ocular irritation, we retrieved 70 patients (127 eyes), who had normal tear production but delayed tear clearance, and did not have clinical evidence of anatomical obstruction at the level of the punctum. The FCT diagnostic criteria for delayed tear clearance are detailed in the Results section, and were established by comparing with that of 20 asymptomatic, age similar, and sex matched patients (40 eyes) who had normal external eye examination and negative dye staining (see Table 1). The criteria were further confirmed by comparing with the tear clearance of another 10 patients (19 eyes) who had punctal obstruction caused by spontaneous closure (five patients), surgical punctal
Accepted for publication 21 January 1998 
occlusion (three patients), or placement of punctal plugs (two patients). An additional inclusion criterion was that all 70 patients had at least two FCT tests - that is, before and after treatment with topical non-preserved $1 \%$ methylprednisolone drops (to be described below). Their clinical data were then retrospectively reviewed under the protocol approved by the Medical Science Subcommittee for the Protection of Human Subjects in Research of the University of Miami School of Medicine.

EXTERNAL EXAMINATION AND TESTS

In general, after history taking, each patient received external examination and the tests described below in the following order. ${ }^{1}$ Tear break up time was measured by adding to the inferior fornix one drop of fluorescein, obtained by wetting on a fluorescein strip (Chauvin Pharmaceuticals Ltd, Harold Hill, Romford, Essex) with non-preserved saline, Unisol (Alcon Laboratories Inc, Fort Worth, TX, USA) in a manner similar to those described by Norn ${ }^{14}$ and Lemp and Hamill. ${ }^{15}$ After a few blinks, the duration in seconds was measured before the precorneal fluorescein tear film broke up. The mean for a total of three times of measurements and the pattern of the film break up were recorded. ${ }^{2}$ Fluorescein staining was immediately evaluated followed by rose bengal staining proceeded by adding $1 \%$ rose bengal solution, either of a commercial source (Akorn Inc, Abita Springs, LA, USA) or prepared by the pharmacy of the Bascom Palmer Eye Institute, onto the superior bulbar conjunctiva without direct contact. After a brief rinse with Unisol, the pattern of staining was recorded. ${ }^{3}$ Biomicroscopic examination was directed to lids, lashes, puncta, tarsal and bulbar conjunctiva, and the cornea. ${ }^{4}$ Finally, FCT was performed after measurement of intraocular pressures and dilatation of pupils.

\section{FLUORESCEIN CLEARANCE TEST}

This test has been briefly described in our previous reports ${ }^{16}{ }^{17}$ and resembles that recently reported by $\mathrm{Xu}$ et al..$^{18}$ In detail, after applying one drop of $0.5 \%$ proparacaine (proxymetacaine) (Alcon Laboratories, Inc, Humacao, PR, USA) to each eye, the inferior fornix was carefully dried with tissue paper. An aliquot of $5 \mu \mathrm{l}$ of Fluoress (Akorn Inc, Abita Springs, LA, USA) containing $0.25 \%$ fluorescein and $0.4 \%$ benoxinate hydrochloride was then applied to the inferior fornix of each eye through an Eppendorf pipette (Rainin Instrument Co, Woburn, MA, USA) without directly touching the conjunctival surface. The patient continued to sit in the examination room under the ambient light and was asked to blink normally. After a lapse of 5 minutes, determined by a timer, a Schirmer paper strip (Alcon Laboratories, Inc, Fort Worth, TX, USA) was inserted into the inferior fornix of each eye at a position approximately one third lateral to the temporal caruncle. After 1 minute, during which time normal blinking was allowed, the strip was removed. The manoeuvre was repeated for a total of six times over a period of 30 minutes. As will be shown in the Results section and discussed in the Discussion section, this test has been simplified by inserting three sets of Schirmer strips-that is, one set for each 10 minutes.

NON-PRESERVED METHYLPREDNISOLONE TREATMENT

All 70 patients received topical treatment with $1 \%$ non-preserved methylprednisolone, which was routinely formulated in our pharmacy by diluting the intravenous preparation of Solumedrol (UpJohn, Kalamazoo, MI, USA) with normal saline. The standard regimen was one drop to each eye three times a day for 3 weeks. Written and verbal instructions were given to advise patients to refrigerate this medication between uses and to avoid direct contact of the dropper with the eye or skin during application. All patients then returned for a follow up visit, during which time subjective changes of symptoms would be graded as improved, worsened, or without any notable difference by comparison with those of the baseline visit. This was followed by a routine eye examination and tests of tear break up time, tension, and FCT as described above.

\section{STATISTICAL ANALYSIS}

With the assistance of the biostatistics department of the Bascom Palmer Eye Institute, demographic data, past history of previous treatments, symptoms, signs of external examination, results of objective tests, and subjective and objective responses to the nonpreserved methylprednisolone treatment were analysed by the $\chi^{2}$ test and Fisher's exact test for categorical variables, and $t$ test and analysis of variance followed by the Student-NewmanKeuls multiple rank test for continuous variables.

\section{Results}

FCT DIAGNOSTIC PATTERNS

Based on our previous experience, we have arrived at the following diagnostic criteria. Tear secretion is regarded normal if the wetting length is equal to or greater than $3 \mathrm{~mm}$; clearance is regarded normal if dye clears after 15 minutes. Figure 1 illustrates the representative FCT patterns. For patients with normal tear secretion and clearance (Fig la and b), each strip showed a wetting length equal to or greater than $3 \mathrm{~mm}$, and the intensity of fluorescein dye fades with time under the blue light and becomes invisible to the naked eye by 15 minutes (that is, the third pair of strips). Towards the intervals of 20 to 25 minutes later (that is, the fourth and fifth pairs of strips), the wetting length was increased because of waning of topical anaesthetics. If this did not happen, the wetting length could be further increased at the final interval of 30 minutes (that is, the sixth pair of strips) by nasal stimulation (not shown). Compared with this normal pattern, patients with decreased aqueous tear production (Fig 1c and d) showed a wetting length of $0 \mathrm{~mm}$-that is, shorter than 3 $\mathrm{mm}$, in all six pairs of strips. Furthermore, the severity was further reflected by an apparent loss of reflex tearing upon nasal stimulation as 

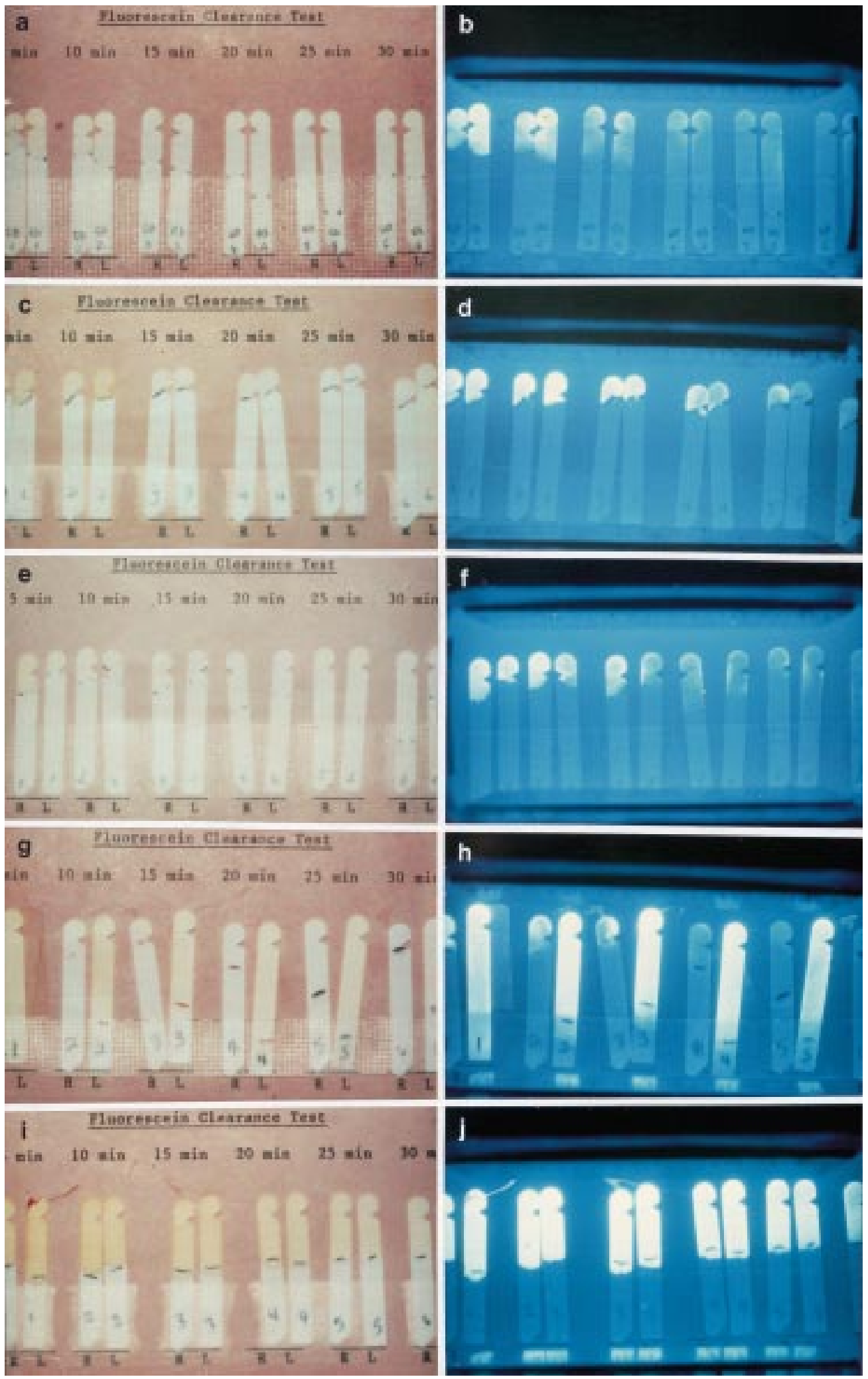

Figure 1 Representative fluorescein clearance test (FCT) results of normal tear secretion and clearance $(a, b)$, severe aqueous tear deficiency $(c, d)$, mild aqueous tear deficiency $(e, f)$, unilateral (left eye) delayed tear clearance $(g, h)$, and bilateral delayed tear clearance $(i, j)$. For each case, six sets of Schirmer paper strips were used in a period of 30 minutes, with one set per 5 minute interval. For each set, the first and second strip was obtained from right and left eye, respectively. The detail of how FCT was performed is given in the Methods section. The entire six sets of strips were taped onto the record card with the wetting length marked by pencil (left panel), and the fluorescein intensity can be better appreciated when photographed under blue light (right panel). 
Table 1 Demographic data of patients with delayed tear clearance

\begin{tabular}{llll}
\hline & $\begin{array}{l}\text { Delayed tear clearance } \\
\text { without apparent } \\
\text { obstruction }\end{array}$ & $\begin{array}{c}\text { Delayed tear clearance } \\
\text { with punctal obstruction }\end{array}$ & $\begin{array}{l}\text { Asymptomatic } \\
\text { normal }\end{array}$ \\
\hline $\begin{array}{l}\text { Number of patients } \\
\text { Number of eyes }\end{array}$ & 70 & 10 & 20 \\
Age (mean (SD)) & 127 & 19 & 40 \\
Sex & $71.4(11.2)$ & $67.9(13.1)$ & $62.8(17.8)$ \\
$\quad$ Male & $24(34 \%)$ & $1(10 \%)$ & $6(30 \%)$ \\
$\quad$ Female & $46(66 \%)$ & $9(90 \%)$ & $14(70 \%)$ \\
$\begin{array}{l}\text { Involvement } \\
\text { Bilateral }\end{array}$ & $57(81 \%)$ & $9(90 \%)$ & $\mathrm{NA}$ \\
$\quad$ Unilateral & $13(19 \%)$ & $1(10 \%)$ & \\
\hline
\end{tabular}

The definition of each group is detailed in the text.

NA $=$ not applicable.

Table 2 Comparison of ocular irritative symptoms

\begin{tabular}{lll}
\hline Symptoms & $\begin{array}{l}\text { Delayed tear clearance } \\
\text { without apparent } \\
\text { obstruction }\end{array}$ & $\begin{array}{l}\text { Delayed tear } \\
\text { clearance with } \\
\text { punctal obstruction }\end{array}$ \\
\hline Tearing & $59 \%$ & $80 \%$ \\
Itching & $26 \%$ & $50 \%$ \\
Mucous discharge and lids stuck together & $26 \%$ & $10 \%$ \\
Redness & $22 \%$ & $20 \%$ \\
Burning & $26 \%$ & $20 \%$ \\
FB sensation & $22 \%$ & $10 \%$ \\
Vague irritation $^{\star}$ & $16 \%$ & $20 \%$ \\
Decreased vision & $10 \%$ & $10 \%$ \\
Photophobia & $10 \%$ & $10 \%$ \\
Miscellaneous & $23 \%$ & $20 \%$
\end{tabular}

The percentage of each complaint is calculated among 70 and 10 patients, respectively. Only those with symptoms greater than $5 \%$ are listed here, and those with less than $5 \%$ are pooled as Miscellaneous, which includes pain, fullness, and tiredness.

${ }^{\star}$ For ocular irritation, those complaining of foreign body sensation are shown under FB sensation, but those with ill defined irritation are classified as vague irritation.

evidenced by the lack of increased wetting length at the end point. However, when tear secretion was not as severely reduced, the wetting length would usually be within 1-2 mm, and was increased substantially by reflex tearing upon nasal stimulation (Fig $1 \mathrm{e}$ and $\mathrm{f}$ ). It is noteworthy that dye clearance in the last two situations is also delayed compared with the normal pattern. In contrast, patients with delayed tear clearance but normal tear secretion (Fig $1 \mathrm{~g}$ and $\mathrm{h}$ ) showed in the right eye mild decreased tear secretion similar to that shown in Figure 1e and f. In contrast, each strip of the left eye had a wetting length within the normal range - that is, equal to or greater than $3 \mathrm{~mm}$, but unlike that of the normal pattern, the dye clearance was markedly delayed (Fig $1 \mathrm{~g}$ and $\mathrm{h}$ ). It should be noted that this left eye did not have any evidence of anatomical obstruction at the punctal level (the corresponding external picture is shown in Fig 2a).

To establish the criterion for delayed tear clearance, we also retrospectively retrieved 20 asymptomatic age similar and sex matched subject (Table 1) with normal external examination, and confirmed that the dye was all cleared from the inferior fornix within $15 \mathrm{~min}-$ utes following application as shown in Figure la and $b$. Based on this difference, we thus defined tear clearance as delayed if dye could still be detected by the strip taken at the 20 minute interval (that is, fourth strip) or later. This criterion was further confirmed by examining another 10 age and sex matched patients who had apparent anatomical obstruction at the punctal level as a result of spontaneous closure, surgical punctal occlusion, or placement of punctal plugs (Table 1). The FCT result of one such example, of which the corresponding external picture is shown in Fig 2b, exhibited a delayed clearance (Fig 1i and $j$ ) similar to that shown in Figure 1g and h (left eye). Based on these criteria, it is possible to judge the extent of severity by comparing the intensity and persistence of dye measured by the strip taken at the end point of 30 minutes.

DEMOGRAPHIC DATA

Based on the diagnostic criteria given above, we retrieved 70 patients (127 eyes) with delayed tear clearance but normal tear secretion. Demographic data revealed that their ages were 71.4 (11.2) years (mean (SD)) (Table 1), which was not different from 67.9 (13.1) years $(\mathrm{p}=0.36)$ for the group with punctal obstruction. Although this age range resembled that of the asymptomatic normals $(62.8(17.8))$, there is a slight statistical difference $(p=0.03)$. This result indicated that the problem of delayed tear clearance occurred primarily in older people. Furthermore, female patients were twice as frequently affected as male patients $(\mathrm{p}=0.0001)$. The majority of patients $(78.6 \%)$ had bilateral involvement.

\section{CLINICAL CHARACTERISTICS}

All 70 patients complained of ocular irritative symptoms, and most patients had multiple complaints giving rise to a total of 166 symptoms. As shown in Table 2, the most prevalent symptom was tearing, which was characterised by its episodic occurrence throughout the day, and thus could be distinguished from epiphora (that is, constant excessive tearing). This symptom also took place in eight of the 10 patients with apparent punctal obstruction. Others included mucous discharge with lids stuck together and crusting in the morning, itching, redness, burning, and foreign body sensation, of which the first three presumably had an inflammatory basis. When these inflammatory complaints were arbitrarily grouped together, they became even more common than episodic tearing. In the group

Table 3 Characteristic external signs

\begin{tabular}{ll}
\hline Rosacea & \\
Lid laxity & $26 \%$ \\
Meibomian gland dysfunction & $39 \%$ \\
$\quad$ Non-expressibility of the meibum & \\
Squamous metaplasia of orifices & $43 \%$ \\
Atrophy & $36 \%$ \\
Puncta & $10 \%$ \\
$\quad$ Normal & \\
Swollen & $62 \%$ \\
Narrowed or slit-like opening & $26 \%$ \\
Conjunctival redness & $12 \%$ \\
Preferentially inferior & $82 \%$ \\
Primarily tarsal & $64 \%$ \\
Tarsal & $64 \%$ \\
Papillary respose & \\
Fluorescein staining & $73 \%$ \\
$\quad$ Negative & \\
Diffuse & $58 \%$ \\
Inferior & $16 \%$ \\
Superior & $16 \%$ \\
Rose bengal staining & $3 \%$ \\
Negative & \\
Inferio & $71 \%$ \\
Interpalpebral exposure zone & $16 \%$ \\
Diffuse & $11 \%$ \\
\hline
\end{tabular}

The percentage is calculated among 70 patients. 

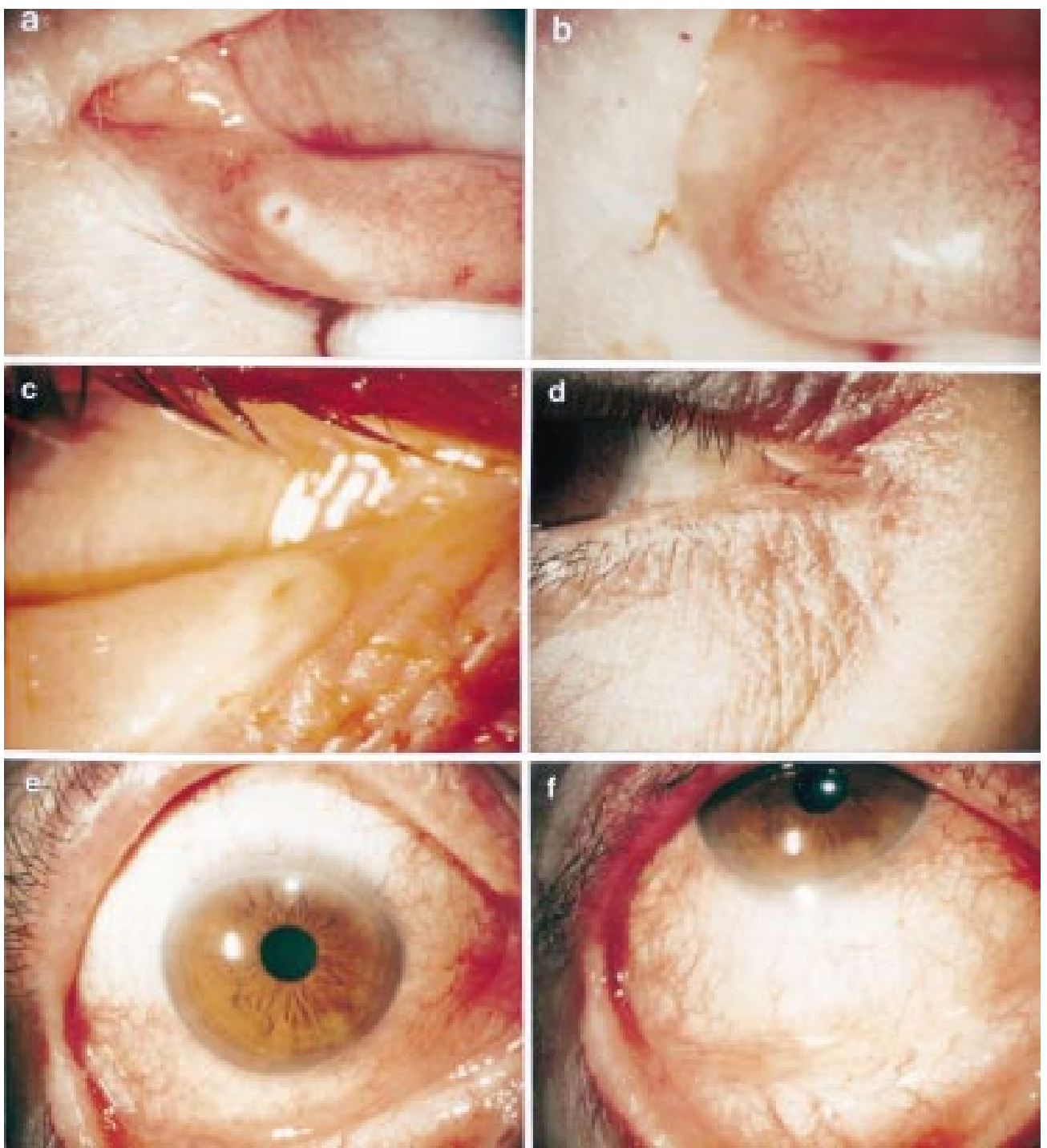

Figure 2 External photographs of a representative case with delayed tear clearance showing no apparent obstruction at the punctum (a). The corresponding fluorescein clearance test (FCT) is shown in Figure 1(g) and (h). For comparison,

another representative case with delayed tear clearance showing punctal obstruction (b), for which the corresponding FCT is shown in Figure 1(i) and (j). The remaining photographs illustrate the typical appearance of a swollen punctum yielding a slit-like appearance (c), the subcutaneous oedema of the adjacent skin (c,d), bulbar conjunctival injection preferentially in the inferior aspect (e), and tarsal conjunctiva with a mild papillary response $(f)$.

with punctal obstruction, 43\% (12/28) was also found to have these inflammatory complaints. When all symptoms were correlated with the diurnal relation, the result showed that $54 \%$ of the above inflammatory symptoms was worse in the morning, $33 \%$ were constant, and $13 \%$ were worse in the afternoon. In the 10 patients with punctal obstruction, we also noted that the above inflammatory symptoms were more frequently worse in the morning $(50 \%)$ than being constant $(34 \%)$ or worse in the afternoon (16\%). Furthermore, $78 \%$ of the symptoms which were worse in the afternoon were burning, foreign body irritation, fluctuating blurred vision, and episodic tearing, as a result of an unstable tear film.

The majority of these patients had been referred and $91 \%$ of them had previously been treated with different kinds of medications under different diagnoses. Among them, artificial tears, commercial preserved steroids, and antibiotics were more commonly used in
$74 \%$ of the patients with negligible improvement. For example, only $15 \%$ of patients receiving commercial preserved steroids actually experienced some short term relief; $23 \%$ of patients receiving other non-steroid treatments experienced limited relief. It was worth noting that $13 \%(9 / 70)$ had a past history of or presented with a clinical picture compatible with medicamentosa. The incidence was even higher-that is, $40 \%(4 / 10)$, in patients with anatomical obstruction at the punctal level. Medicamentosa, when taken as $100 \%$, manifested as diffuse conjunctival redness (89\%) and punctate keratopathy (33\%) (also see case example) and drug induced pseudopemphigoid $(11 \%)$.

We noted that $9 \%$ of these 70 patients were using antiglaucoma medications-five patients had glaucoma, and 11 patients had ocular hypertension before the treatment with methylprednisolone. Among the latter 11 patients, three had never received steroid treatments, 
and the remaining eight patients had received commercial steroids. After the methylprednisolone treatment, eight additional patients developed steroid induced ocular hypertension. Taken together, there was a total of 24 patients $(34.3 \%)$ with glaucoma (five patients, two males, and three females) or ocular hypertension (19 patients, seven males, and 12 females).

Characteristic external signs are summarised in Table 3 and Figure 2. Rosacea, lid laxity, and meibomian gland dysfunctions were common. About two thirds of the patients with lid laxity was found to have floppy eyelids. The skin near the nasal caruncle of the involved eye(s) frequently showed subcutaneous swelling as evidenced by increased wrinkles and being darkened (Fig 2c and d). Puncta frequently showed swelling (Fig 2c) and narrowed opening to slit-like appearance. Conjunctival redness was also common and preferentially in the inferior bulbar and tarsal areas (Fig 2e) and the tarsal greater than bulbar involvement (Fig 2f). Dye staining tended to be negative but if positive the involvement tended to be in the non-exposure zone. Interestingly, those patients with non-exposure zone staining had a
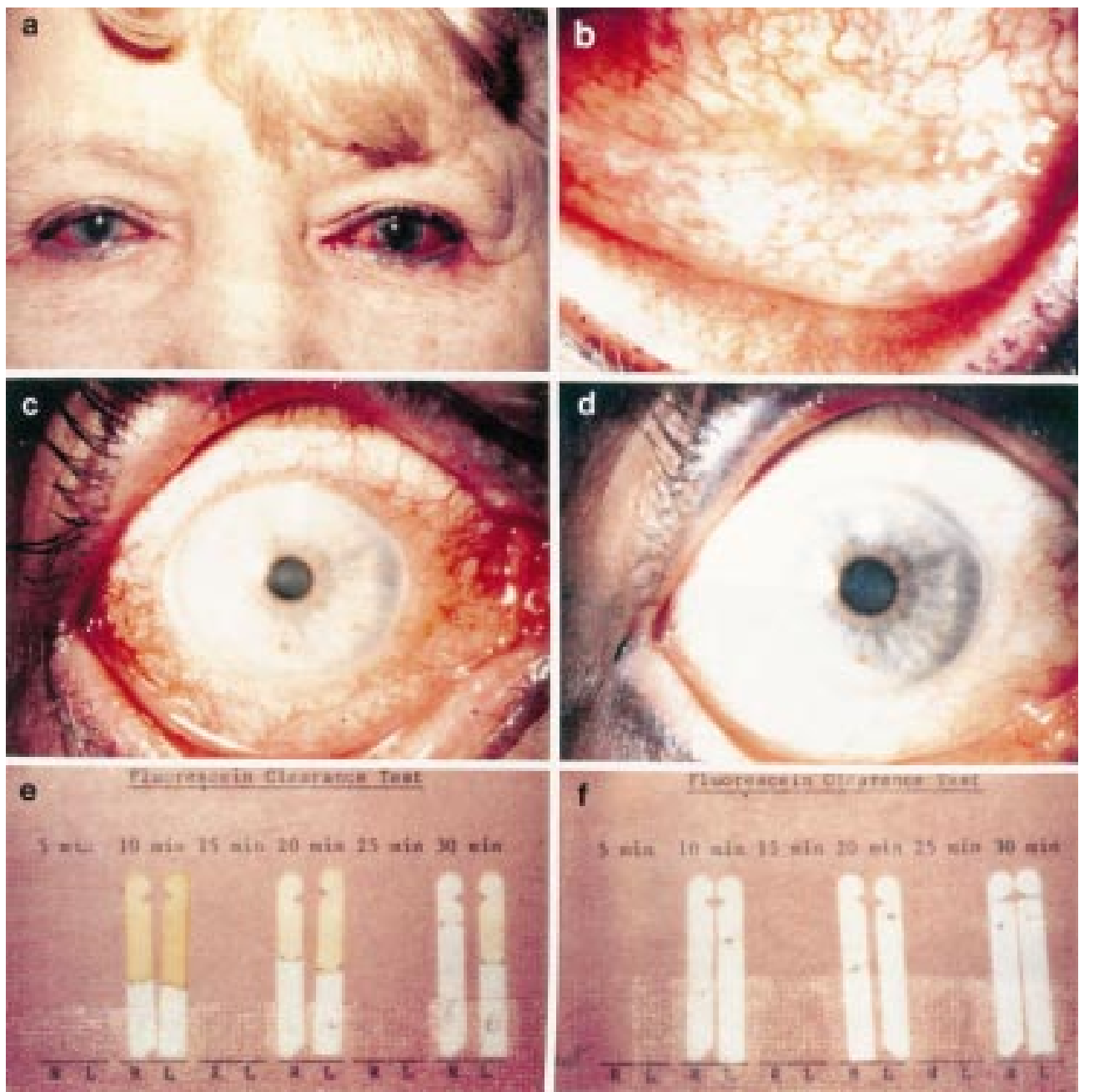

Figure 3 External appearance of case example showing diffuse conjunctival injection of both eyes (a) which was also preferentially distributed in the inferior tarsal conjunctiva with a mixed papillary and follicular response (b) and the entire bulbar conjunctiva (c) before the use of methylprednisolone. After treatment for 2 weeks, the entire bulbar conjunctiva became white and quiet (d). Fluorescein clearance test revealed marked delayed clearance of fluorescein (e), which was markedly improved after treatment $(f)$.

$57 \%$ chance of, whereas those of exposure zone staining had none, expressing inflammatory complaints.

PRESERVATIVE-FREE METHYLPREDNISOLONE

TREATMENT

The tear film was unstable-that is, the tear break up time was shorter than 8 seconds, in $86 \%$ of eyes. The tear break up time was 3.0 (SD 2.9) seconds before the methylprednisolone treatment, but significantly improved to 5.0 (2.9) seconds after the treatment ( $\mathrm{p}$ $<0.001)$. The average duration of nonpreserved $1 \%$ methylprednisolone treatment was 3.0 (1.1) weeks. After the treatment, $83 \%$ of the patients noted subjective improvements with resolution of previous irritative symptoms. This was supported by objective improvements with respect to the resolution of abnormal signs in $80 \%$ of patients. These included reduction of redness, decrease or absence of dye staining, or reduction of tarsal papillary responses. Most importantly, these improvements were also correlated with the result of the FCT test. Of all patients, $87 \%$ showed improvement in dye clearance, $10 \%$ of patients had no change, and $3 \%$ of patients
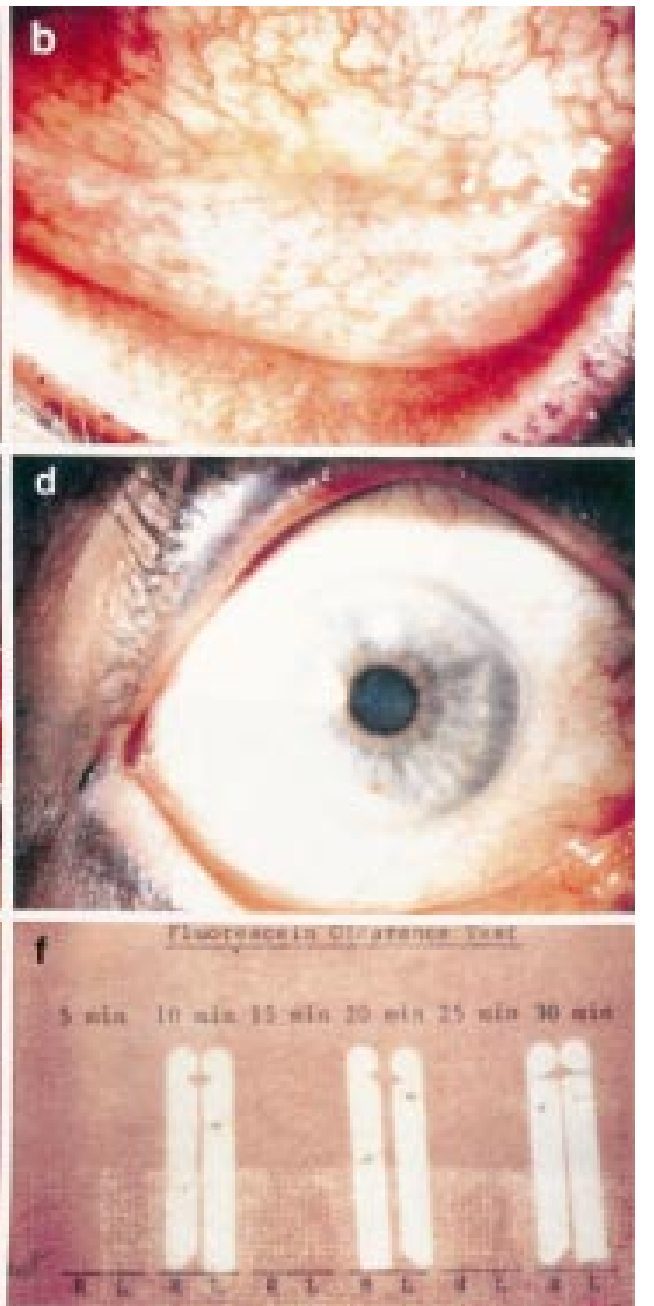
showed worsened clearance. Among those who showed improvement by FCT, $28 \%$ reverted to the normal pattern with normal clearance, $43 \%$ showed improved clearance while retaining the same wetting length as judged by the intensity of dye retained at the last Schirmer strip, and the remaining 30\% showed improved clearance but with reduced wetting length. Two eyes of one patient out of 16 eyes that did not show improvement were later on proved to have anatomical obstruction by probing and irrigation.

Following the cessation of the treatment, 22 patients $(31 \%)$ showed recurrences ranging from one to four times with the mean of 1.6 (0.9) times. The mean duration for recurrence was 9.9 (18.4) months. Among these 22 patients, 17 were retreated with methylprednisolone and all showed improvement. Clinically, these 22 patients were noted to have allergy $(27 \%)$, punctal stenosis (23\%), lid abnormality (18\%), neurotrophic keratopathy $(9 \%)$, and cicatricial conjunctivitis $(5 \%)$, or problems which developed following cataract surgery $(5 \%)$.

\section{CASE EXAMPLE}

A 70 year old woman with a past history of chronic bronchitis complained of episodic tearing for 7 years and conjunctival redness and itching for 5 months. She had been unsuccessfully treated with various topical medications including artificial tears, conventional steroids, and antibiotics, and had been noted to have a patent drainage system with multiple times of probing and irrigation. Upon first presentation (16 March 1988), her vision was best corrected to 20/30 right eye and 20/20 left eye. Her face had telangiectasia and lids were lax with intact closure and blinking. Her tarsal and bulbar conjunctiva were diffusely injected (Fig 3a-c) with tarsal conjunctiva showing a mixed papillary and follicular response (Fig 3b). All four puncta were swollen and collapsed into a slit-like appearance. Tear break up time was greater than 10 seconds (normal) each eye. Rose bengal staining was positive over inferior cornea and bulbar conjunctiva in both eyes and some exposure zones in the right eye. FCT revealed marked delayed dye clearance but normal tear secretion (Fig 3e). Under the impression of allergy with medicamentosa in the context of delayed tear clearance, the patient was treated with non-preserved $1 \%$ methylprednisolone four times a day for 2 weeks together with Unisol irrigation. She noted significant improvement with resolution of conjunctival redness (Fig 3d), and our examination revealed decreased tarsal reaction and improved tear clearance (Fig 3f). Since then, she has experienced four recurrences (24 May 1989, 11 October 1989, 3 June 1990, and 13 March 1991), each time with delayed tear clearance confirmed by FCT, with improvement by resuming methylprednisolone treatment, and with improved clearance confirmed by repetitive FCT.

\section{Discussion}

SUBCLINICAL DELAYED TEAR CLEARANCE CAN BE DETECTED BY FCT

The basis for FCT to detect delayed tear clearance resembles those performed by such pioneering investigators as Nover and Jaeger, ${ }^{3}$ Norn, ${ }^{19}$ Mishima et $a l,{ }^{8}$ and Zappia and Milder ${ }^{7}$ because they all measured the rate of fluorescein disappearance from the eye. Unlike these earlier studies, ${ }^{3719}$ in which the measurement was taken only once at 2 minutes, 5 minutes, and 5 minutes after dye application, respectively, FCT is conducted over a period of 30 minutes. It is important to perform serial measurements over a longer period of time because kinetic studies by fluorophotometry ${ }^{8-13}$ and by serial Schirmer strips ${ }^{20}$ have revealed that there are two phases of fluorescein clearance over a period of 30 minutes. The initial phase, taking place within 5 minutes after dye application, has a rapid clearance rate, and is highly variable among different individuals. ${ }^{82}$ This variability is thought to be caused by stimulation of lacrimation, ${ }^{8}$ and can be suppressed by topical anaesthetics. ${ }^{9}$ Because variability of this initial phase is influenced by the applied volume in both humans ${ }^{89}$ and rabbits $^{22}$ and by factors triggering reflex tearing, ${ }^{93}$ we chose to apply a small volume of $5 \mu \mathrm{l}$ and conducted the test after topical anaesthetics. The second phase, taking place 5 minutes after dye application, is slower but more constant, and has been used by many investigators to calculate the physiological rate of tear flow and clearance (turnover) using fluorophotometry. ${ }^{8-13}$ Because fluorescein concentration is semiquantitated by Schirmer paper strips, FCT might inherit criticism given to the Schirmer test, which is known to have a wide range of false positivity and false negativity in differentiating patients with keratoconjunctivitis sicca from normals. ${ }^{24} 25$ This variability is thought to be derived from the paper's touching eyelashes, eliciting reflex tearing, which cannot be suppressed by topical anaesthetics as suggested by Jordan and Baum. ${ }^{9} \mathrm{We}$ thus chose to insert the Schirmer strip for only 1 minute instead of 5 minutes so as to minimise this potential error. Although we realise that fluorescein measurement by FCT is not as accurate as fluorophotometry, we are reassured by the result of Holly et $a{ }^{20},{ }^{20}$ showing that the wetting length of Schirmer strips can be semiquantitative. Recently, this measurement of tear clearance has been found to be consistent with that obtained from fluorophotometry. ${ }^{26}$ FCT provides an additional advantage that all materials used are readily available in all clinical settings. Based on our experience and data shown in Figure 1, we can use FCT to detect tear clearance besides (basal) tear secretion under anaesthetics and reflex tearing under nasal stimulation.

It should be noted that all these 70 patients did not have apparent anatomical obstruction at the punctal level, and that the majority did not have anatomical obstruction in the nasolacrimal drainage system. The latter notion is supported by the facts that $4 \%(3 / 70)$ of them had a patent passage proved by prior probing 
and irrigation, that tear clearance improved in the majority of patients ( $87 \%$ ) simply by nonsurgical methylprednisolone treatment, and that only $7 \%(1 / 14)$ of those who did not improve after the treatment were later on found to have anatomical obstruction by probing and irrigation. In addition, delayed tear clearance has been observed in so called "normals"- that is, without frank epiphora, using traditional dye disappearance test. ${ }^{727}$ Collectively, these findings indicate that subclinical delayed tear clearance is present and might have generally been overlooked

CLINICAL CHARACTERISTICS OF DELAYED TEAR CLEARANCE

From the outset, these 70 patients appeared to have complex symptomatology, and their past ocular history also revealed multiple diagnoses and different treatments. Demographically, the majority of them were older women. When grouped together, the most common complaints are of an inflammatory nature such as itching, redness, and mucus discharge with lids stuck together, which tend to be worse in the morning upon awakening. The second common symptom is episodic tearing, which is potentially a result of excessive tear production but aggravated by the underlying delayed tear clearance. These symptoms have been reproduced by blocking one of the two canaliculi with a punctal plug in normal volunteers and then by exposing them to a stressful environment, such as Colorado high mountains. ${ }^{28}$ Other common symptoms, including burning, foreign body sensation, and fluctuating blurred vision, most likely result from an unstable tear film because most patients had a short tear break up time. We thus speculate that it is the unstable tear film triggering the reflex tearing and allowing episodic tearing to manifest as a result of the underlying delayed tear clearance.

We speculate that their clinical characteristics can be explained by the common denominator of delayed tear clearance although differences in tear contents and other analyses might potentially be involved. For tears to be cleared from the ocular surface, clearance via the nasolacrimal drainage system is far more important than absorption by the ocular surface ${ }^{29}$ and than evaporation. ${ }^{30}$ Several image studies have confirmed that the nasolacrimal drainage system relies on lid blinking to generate a pumping effect to draw tears into the lacrimal sac in rabbits ${ }^{31}$ and in humans..$^{32-34}$ This may explain why lax and floppy lids found in our patients might contribute to the development of delayed tear clearance. Furthermore, it has been noted that corneal sensitivity progressively decreases with age, ${ }^{35}$ and that decreased sensitivity is the major cause of decreased blinking. ${ }^{37}$ Blinking is also known as the key factor controlling tear clearance ${ }^{929} 31$ especially during the initial rapid phase. ${ }^{23}$ Fluorophotometric studies have also shown that older people, especially women, tend to lose the initial phase of rapid clearance and have a slower tear clearance rate. ${ }^{8}$ Taken together, these data explain why older patients, especially women, are more susceptible to this abnormality. Because blinking ceases during sleep, ${ }^{38}$ thus leading to further delayed tear clearance, ${ }^{23} 33$ this may explain why such patients frequently complained that their ocular irritation was much worse in the morning upon awakening. Because tear clearance is facilitated by gravitation, ${ }^{33}$ this also explains why delayed tear clearance precipitated a preferential involvement of the inferior bulbar and tarsal conjunctiva with respect to the redness in medicamentosa (Figs 2 and 3) and cicatricial changes in drug induced pseudopemphigoid. The fact that some patients were prone to develop medicamentosa manifesting as diffuse redness, superficial punctate keratopathy (case example), drug induced pseudopemphigoid, or ocular hypertension can be attributed to the use of topical medications such as preservativecontaining medications including tears, antibiotics, antiglaucoma drops, and steroids. ${ }^{39} 40$ Because of the underlying delayed tear clearance, these medications clear much more slowly from the eye, resulting in a relatively higher concentration and a longer transit time on the ocular surface, a concept proved by the use of fluorophotometry. ${ }^{16}$ It is worth noting that the percentage of patients in this group suffering from ocular hypertension $(27.2 \%)$ or glaucoma $(7.2 \%)$ is significantly greater than the reported average of $10.5 \%$ and $2 \%$, the prevalence of ocular hypertension and open angle glaucoma, respectively, in geriatric populations (age 70 to 79 ). ${ }^{41}$ Furthermore, the tear clearance rate is decreased in patients with untreated primary open angle glaucoma. ${ }^{42}$ Future studies are needed to determine if delayed tear clearance might contribute to the high prevalence of steroid induced ocular hypertension.

In addition to infrequent and ineffective blinking as described above, delayed tear clearance can also be aggravated by a compromised, although not completely obstructed, drainage system. This is evidenced by swollen and slit-like punctal openings noted in some patients (Fig 2). This pathology can be found in patients with allergy as proposed by others, ${ }^{43}$ rosacea, or inflammatory meibomian gland dysfunction. It can also be caused by toxic effects from topical medications, of which preservatives, miotics, and certain antibiotics have been implicated. ${ }^{39}{ }^{40}$ Taken together, it is likely that medicamentosa can precipitate delayed tear clearance, which in turn can perpetrate medicamentosa in a vicious cycle. We believe that this vicious cycle can be broken by topical non-preserved methylprednisolone. This therapeutic effect can be explained by the steroidal anti-inflammatory action, which reduces mucosal swelling in the canalicular system leading to improved tear clearance. We also attribute this efficacy to its preservative-free nature because $16 \%$ of patients who had previously used commercial preserved steroids actually experienced a short term effect. We further noted that some of them actually became worse upon prolonged use of preserved steroids. As a contrast, the majority of the 70 patients who received non-preserved 


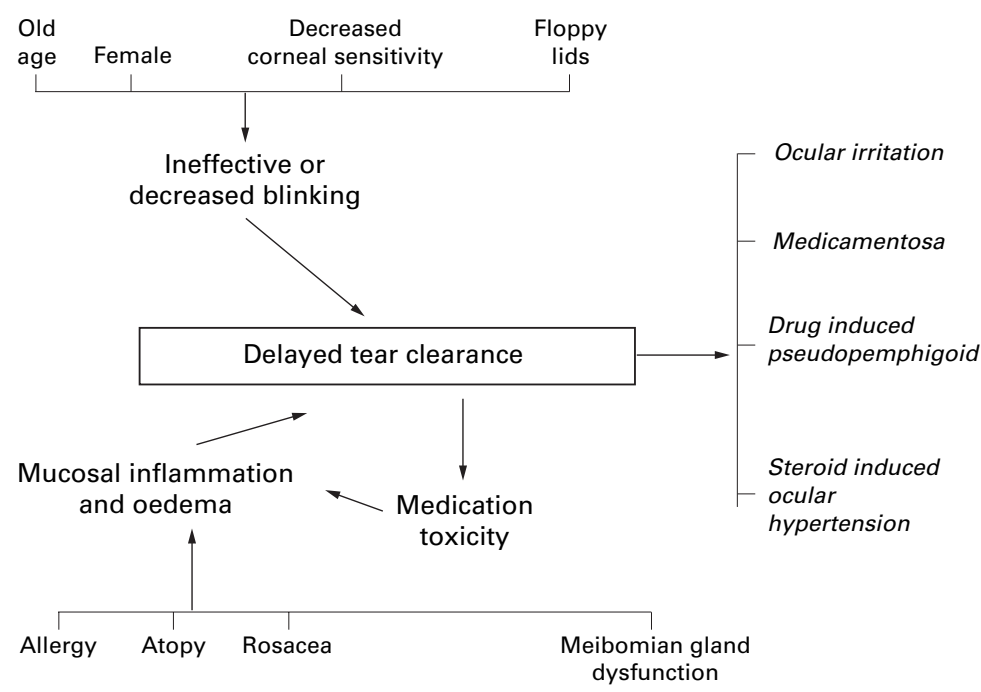

Figure 4 Hypothetical scheme of the pathophysiology of delayed tear clearance. Delayed tear clearance can be induced by ineffective or decreased blinking (functional block) or mucosal inflammation and oedema in the nasolacrimal drainage system (partial anatomical block). The former is contributed by such risk factors as old age, female sex, decreased corneal sensitivity, and lid laxity including floppy lids. The latter can intrinsically be generated by allergy, atopy, rosacea, and meibomian gland dysfunction, or extrinsically caused by medication toxicity. $A$ vicious cycle can form between delayed tear clearance and mucosal inflammation and oedema leading to ocular irritation, medicamentosa, drug induced pseudopemphigoid, and steroid induced ocular hypertension.

methylprednisolone showed subjective (83\%) and objective $(80 \%)$ improvements, and resolution of delayed tear clearance by FCT $(87 \%)$. Furthermore, such an improvement had a lasting effect because recurrence took place after a mean of 10 months later. Moreover, each recurrence was confirmed by repetitive FCT showing delayed tear clearance, and could be resolved after repeated treatment (see case example). Thus, we wonder whether preservatives in topical medications might in general impose an adverse effect on the ocular surface whenever there is delayed tear clearance.

Although we demonstrate here that there is strong association of delayed tear clearance with a certain form of ocular irritation, and that resolution of delayed tear clearance correlates well with clinical improvement, we cannot conclude its causative role even if such a role has been implied in the study using normal volunteers. ${ }^{28}$ Nevertheless, we speculate that once delayed tear clearance has set in, it can perpetuate the development of mucosal inflammation through a vicious cycle especially in those susceptible patients. Recent evidence suggests that patients with delayed tear clearance frequently had a significantly higher concentration of interleukin 1 in their tears. ${ }^{44} \mathrm{It}$ should be noted that even simple closed eye during sleep is known to generate inflammation in normal individuals. ${ }^{45}$ Therefore, it is likely that delayed tear clearance, even without knowing whether it has a direct causative role, can aggravate either nocturnally or pathologically occurring ocular inflammation. Therefore, we believe that future studies are warranted to delineate the pathogenic role of delayed tear clearance. Our hypothetical view is summed up in Figure 4, and can be used by others to examine its accuracy.
Proprietary interest: The use of non-preserved methylprednisolone for the treatment of delayed tear clearance has been filed for US patent protection by the University of Miami.

Supported in part by Public Health Service Research Grant No EY 10900 from Department of Health and Human Services, National Eye Institute, National Institutes of Health, Bethesda, Maryland, and in part by an unrestricted grant from Research to Prevent Blindness, Inc, New York, New York, USA.

1 Holly FJ, Lemp MA. Tear physiology and dry eyes. Surv Ophthalmol 1977;22:69-87.

2 Lamberts DW. Physiology of the tear film. In: Smolin G, Thoft RA, eds. The cornea: scientific foundations and clinical practice. Boston/New York/Toronto/London: Little Brown, 1994:439-56.

3 Nover A, Jaeger W. Kolorimetrische Methode zur Messung der Tranensekretion (Fluoreszein-Verdunnungstest). Klin Monatsbl Augenheilkd 1952;121:419-25.

4 Jones LT. The lacrimal secretory system and its treatment. Am F Ophthalmol 1966;62:47-60.

5 Tucker NA, Codere F. The effect of fluorescein volume on lacrimal outflow transit time. Ophthal Plast Reconst Surg 1994;10:256-9.

6 Norn MS. Tear secretion in normal eyes. Estimated by a new method: the lacrimal streak dilution test. Acta new method: the lacrima

7 Zappia RJ, Milder B. Lacrimal drainage function. 2. The fluorescein dye disappearance test. Am F Ophthalmol 1972; $74: 160-2$

8 Mishima S, Gasset A, Klyce SDJ, et al. Determination of tear volume and tear flow. Invest Ophthalmol 1966;5:26476.

9 Jordan A, Baum J. Basic tear flow. Does it exist? Ophthalmology 1980;87:920-30.

10 Puffer MJ, Neault RW, Brubaker RF. Basal precorneal tear turnover in the human eye. Am F Ophthalmol 1980;89:36976.

11 Webber WRS, Jones DP, Wright P. Fluorophotometric measurements of tear turnover rate in normal healthy persons: evidence for a circadian rhythm. Eye 1987;1:61520 .

12 Occhipinti JR, Mosier MA, LaMotte J, et al. Fluorophotometric measurement of human tear turnover rate. Curr Eye metric measurement of

13 Van Best JA, Benitez del Castillo JM, Coulangeon L. Measurement of basal tear turnover using a standardized protocol. European concerted action on ocular fluorometry. Graefes Arch Clin Exp Ophthalmol 1995;233:1-7.

14 Norn MS. Desiccation of the precorneal film. I. Corneal wetting-time. Acta Ophthalmol 1969;47:865-80.

15 Lemp MA, Hamill JR. Factors affecting tear film breakup in normal eyes. Arch Ophthalmol 1973;89:103-5.

6 Linden C, Alm A. The effect of reduced tear drainage on corneal and aqueous concentrations of topically applied fluorescein. Acta Ophthalmol 1990;68:633-8.

17 Pflugfelder SC, Tseng SCG, Pepose JS, et al. Epstein-Barr virus infection and immunological dysfunction in patients with aqueous tear deficiency. Ophthalmology 1990;97:313-
23 .

$8 \mathrm{Xu} \mathrm{K}$, Yagi Y, Toda I, et al. Tear function index. A measure of dry eye. Arch Ophthalmol 1995;113:84-8.

19 Norn MS. Lacrimal apparatus test. A new method (lacrimal streak dilution test) compared with previous methods. Acta Ophthalmol 1965;43:557-566 (abstract).

20 Holly FJ, Lamberts DW, Esquivel ED. Kinetics of capillary tear flow in the Schirmer strip. Curr Eye Res 1983;2:57-70.

21 Kuppens EVJ, Stolwijk TR, de Keizer RJW, et al. Basal tear turnover and topical timolol in glaucoma patients and healthy controls by fluorophotometry. Invest Ophthalmol Vis Sci 1992;33:3442-8.

22 Chrai SS, Patton TF, Mehta A, et al. Lacrimal and instilled fluid dynamics in rabbit eyes. F Pharm Sci 1973;62:111221.

23 White WL, Glover AT, Buckner AB. Effect of blinking on tear elimination as evaluated by dacryoscintigraphy. Ophthalmology 1991;98:367-9.

24 Bijsterveld OP. Diagnostic tests in sicca syndrome. Arch Ophthalmol 1969;82:10-14.

25 Cho P, Yap M. Schirmer test. I. A review. Optom Vis Sci 1993;70:152-6.

$26 \mathrm{Xu}, \mathrm{K}$ and Tsubota $\mathrm{K}$. Correlation of tear clearance rate and fluorophotometric assessment of tear turnover. $\mathrm{Br} \mathcal{f}$ Ophthalmol 1995;79:1042-5.

27 Meyer DR, Antonello A, Linberg JV. Assessment of tear drainage after canalicular obstruction using fluorescein dye disappearance. Ophthalmology 1990;97:1370-4.

28 Reed S, Lissner G. Clinical study on the effectiveness of tear drainage with a single canalicular system under environmental stress. Ophthal Plast Reconstr Surg 1993;9:27-31.

29 Webber WR, Jones DP. Continuos fluorophotometric method of measuring tear turnover rate in humans and analysis of factors affecting accuracy. Med Biol Eng Comput 1988;24:386-92.

30 Tomlinson A, Trees GR, Occhipinti JR. Tear production and evaporation in the normal eye. Ophthal Physiol Opt 1991;11:44-7.

31 Patton TF, Robinson JR. Influence of topical anesthesia on tear dynamics and ocular drug bioavailability in albino rabbits. F Pharm Sci 1975;64:267-71.

32 Doane MG. Blinking and the mechanics of the lacrimal Doane MG. Blinking and the mechanics of the
drainage system. Ophthalmology 1981;88:844-51.

33 Fraunfelder FT. Extraocular fluid dynamics: how best to apply topical ocular medication. Trans Am Ophthal Soc 1976;74:457-87. 
34 Lemp MA, Weiler HH. How do tears exit? Invest Ophthalmol Vis Sci 1983;24:619-22.

35 Boberg-Ans J. On the corneal sensitivity. Acta Ophthalmol 1956;34:149-62.

36 Millodot $M$. The influence of age on the sensitivity of the cornea. Invest Ophthalmol Vis Sci 1977;16:240-2.

37 Collins $\mathrm{M}$, Seeto R, Campbell L, et al. Blinking and corneal sensitivity. Acta Ophthalmol 1989;67:525-31.

38 Hoshina Y, Sakuma Y. Changes in phototically evoked blink reflex during sleep and wakefulness. Fpn $\mathcal{F}$ Ophthalmol 1991;35:182-7.

39 Burstein NL. Corneal cytotoxicity of topically applied drugs, vehicles and preservatives. Surv Ophthalmol 1980; 25:15-30.

40 Fraunfelder FT, Meyer SM. Corneal complications of ocular medications. Cornea 1986;5:55-9.
41 Hart JWM. The epidemiology of primary open-angle glaucoma and ocular hypertension. In: Ritch R, Shields MB, Krupin T. eds. The glaucomas. St Louis: CV Mosby, 1989: $789-95$.

42 Kuppens EVM, van Best JA, Sterk CC, et al. Decreased basal tear turnover in patients with untreated primary open-angle glaucoma. Am f Ophthalmol 1995;120:41-6.

43 Wojno TH. Allergic lacrimal obstruction. Am f Ophthalmol 1988;106:48-52.

44 Barton K, Monroy DC, Nava A, Pflugfelder SC. Inflammatory cytokines in tears of patients with ocular rosacea. $O p h$ thalmology 1997;104:1868-74.

45 Sack RA, Tan KO, Tan A. Diurnal tear cycle: evidence for a nocturnal inflammatory constitutive tear fluid. Invest $O p h$ thalmol Vis Sci 1992;33:626-40. 